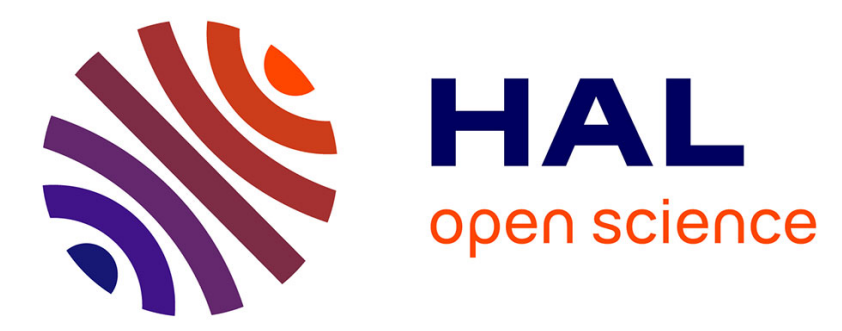

\title{
Motor urgency is mediated by the contralateral cerebellum in Parkinson's disease
}

B Ballanger, Pierre Baraduc, E. Broussolle, D. Le Bars, M. Desmurget, S.

Thobois

\section{- To cite this version:}

B Ballanger, Pierre Baraduc, E. Broussolle, D. Le Bars, M. Desmurget, et al.. Motor urgency is mediated by the contralateral cerebellum in Parkinson's disease. Journal of Neurology, Neurosurgery and Psychiatry, 2008, 79 (10), pp.1110-1116. 10.1136/jnnp.2007.141689 . hal-02128274

\section{HAL Id: hal-02128274 \\ https://hal.science/hal-02128274}

Submitted on 14 May 2019

HAL is a multi-disciplinary open access archive for the deposit and dissemination of scientific research documents, whether they are published or not. The documents may come from teaching and research institutions in France or abroad, or from public or private research centers.
L'archive ouverte pluridisciplinaire HAL, est destinée au dépôt et à la diffusion de documents scientifiques de niveau recherche, publiés ou non, émanant des établissements d'enseignement et de recherche français ou étrangers, des laboratoires publics ou privés. 


\title{
Motor urgency is mediated by the contralateral cerebellum in Parkinson's disease
}

\author{
B Ballanger, ${ }^{1}$ P Baraduc, ${ }^{1}$ E Broussolle, ${ }^{2}$ D Le Bars, ${ }^{3}$ M Desmurget, ${ }^{1}$ S Thobois ${ }^{2}$
}

${ }^{1}$ Center of Cognitive Neuroscience, CNRS UMR 5229, Bron, France; ${ }^{2}$ INSERM, U 864, Bron, France; ${ }^{3}$ CERMEP Imagerie du vivant, PET Unit, Lyon, France

Correspondence to: Dr S Thobois, Service de Neurologie C, Hôpital Neurologique Pierre Wertheimer, 59 Bd Pinel, 69003 Lyon, France; stephane.thobois@chu-lyon.fr

Received 5 December 2007 Revised 11 February 2008 Accepted 10 March 2008 Published Online First 20 March 2008

\begin{abstract}
Background: In patients with Parkinson's disease (PD), motor performance may be dramatically improved in urgent and stressful situations.

Objective: The aim of this PET $\mathrm{H}_{2}{ }^{15} \mathrm{O}$ study was to determine the changes in brain activation pattern related to this unconscious increase in motor speed observed in the context of urgency in patients with PD.
\end{abstract}

Methods: Eight right-handed patients with PD, who had been off medication for at least 12 hours, without tremor, were enrolled. A reaching task with the right hand was performed under three conditions: self-initiated (SI), externally cued (EC) and externally cued-urgent (ECu). Results: (1) Self-initiated movements (SI-EC) revealed activations in the prefrontal cortex bilaterally, the right lateral premotor cortex, anterior cingulate cortex and cerebellum, and the left primary motor cortex and thalamus; (2) Externally driven responses (EC-SI) did not involve any statistically detectable activation; (3) Urgent situations (ECu-EC) engaged the left cerebellum. Compared with a control group previously studied, the cerebellar activation was greater in patients with PD.

Conclusions: This study demonstrates that the increase in movement speed in urgent situations in patients with $\mathrm{PD}$ is associated with the recruitment of the left (contralateral) cerebellum. This structure is a key node of the accessory motor circuitry typically recruited by patients with PD to compensate for basal ganglia dysfunction and by healthy subjects to increase movement velocity in urgent motor contexts.

Bradykinesia is a cardinal clinical symptom of Parkinson's disease (PD). During the past 15 years, converging observations have suggested that this symptom could be overcome in some circumstances. In particular, it was reported that patients with PD exhibited bradykinesia when performing reaches to a stationary ball or a visual target, but not when reaching for a moving ball or when pressed to move fast. ${ }^{12}$ In the same vein, it was found that patients with $\mathrm{PD}$ did perform sequential arm movements significantly faster in the presence of external temporal cues. ${ }^{3}{ }^{4}$ To account for these effects, it was suggested that different neural networks were involved in the control of self-initiated and externally cued movements. ${ }^{5-7}$ In agreement with this view, functional imaging studies, in PD, identified activation within the basal ganglia for self-initiated but not for externally cued movements. ${ }^{8} 9$ Other reports identified a relationship between the specific recruitment of a cerebellar-parieto-premotor circuitry and the improvement of motor performance by external cues. ${ }^{10-13}$ Recently, however, the existence of different neural networks for self-initiated and externally cued movements was questioned based on the existence of contextual variations of movement duration in healthy subjects. ${ }^{14}$ These contextual variations were found in controls to rely on the recruitment of an accessory motor pathway involving the anterior (ie, uncrossed) corticospinal tract (ipsilateral sensorimotor cortex) and the contralateral cerebellum. ${ }^{15}$

To our knowledge, no functional imaging study has investigated directly the modifications of brain activation related to motor urgency in $\mathrm{PD}$. The present PET $\mathrm{H}_{2}{ }^{15} \mathrm{O}$ study aims to fill this gap.

\section{METHODS}

\section{Subjects}

Eight patients with $\mathrm{PD}$ with bilateral akinetic-rigid signs ( 7 males, 1 female; mean age $62 \pm 6$ years) participated in the study (table 1). All patients were right-handed and fulfilled the UK Parkinson's Disease Brain Bank criteria for idiopathic PD. ${ }^{16}$ They did not exhibit severe tremor in the Off condition. On the day of the PET scan, they were off antiparkinsonian drugs for at least 12 hours and the severity of parkinsonian symptoms was assessed using the Unified Parkinson's Disease Rating Scale (UPDRS) motor score. ${ }^{17}$

The study was approved by the local research ethics committee. All patients participated after the aims of the study and the nature of the procedures had been fully explained. They signed an informed consent form according to the Declaration of Helsinki.

\section{Tasks}

The behavioural apparatus and tasks have been described in detail elsewhere. ${ }^{14}$ In brief, subjects lay supine on the scanner bed with the right hand in contact with a press button positioned in the middle of the abdomen in a relaxed posture $40 \mathrm{~cm}$ away from a large vertical contact plate $(28 \times 18 \mathrm{~cm})$ suspended over the scanner bed. Contact with this plate controlled an electromagnetic catch located at the bottom of a tilted ramp. Patients were instructed to maintain gaze fixation on a bright green dot positioned in front of the electromagnetic catch. The apparatus is illustrated in figure 1.

Three types of conditions were considered:

1. Self-Initiated (SI). The patients were required to "wait until they felt ready and then hit the push button with the right hand with the fastest possible movement". Just after completion of the movement, an auditory beep was presented (identical to the beep presented in the externally cued conditions) and the green light switched off, instructing the subjects to 
jn141689 Module 1 Journal of Neurology, Neurosurgery, and Psychiatry 22/7/08 17:32:16

Research paper

Table 1 Clinical characteristics of patients with Parkinson's Disease

\begin{tabular}{lllllll}
\hline Patients & Sex & Age (years) & H \& Y (Off) & Motor UPDRS (Off) (108) & $\begin{array}{l}\text { Disease duration } \\
\text { (years) }\end{array}$ & Medication in Ldopa equivalent (mg/day) \\
\hline 1 & $\mathrm{~F}$ & 58 & 2 & 26 & 29 & 600 \\
2 & $\mathrm{M}$ & 65 & 3 & 33 & 20 & 1050 \\
3 & $\mathrm{M}$ & 66 & 2 & 18 & 8 & 750 \\
4 & $\mathrm{M}$ & 66 & 2 & 26 & 9 & 750 \\
5 & $\mathrm{M}$ & 56 & 3 & 28 & 10 & 1150 \\
6 & $\mathrm{M}$ & 71 & 2 & 18 & 8 & 1050 \\
7 & $\mathrm{M}$ & 57 & 3 & 31 & 11 & 1300 \\
8 & $\mathrm{M}$ & 58 & 2 & 24 & $13 \pm 7$ & 1750 \\
Mean $\pm S D$ & & $62 \pm 6$ & $2 \pm 1$ & $26 \pm 5$ & $1050 \pm 367$
\end{tabular}

F, Female; H \& Y, Hoehn and Yahr; Ldopa equivalent, $100 \mathrm{mg} \mathrm{L-dopa} \mathrm{=} 10 \mathrm{mg}$ Bromocriptine = $5 \mathrm{mg}$ Ropinirole = $1 \mathrm{mg}$ Pergolide = $50 \mathrm{mg}$ Piribedil; M, Male; SD, Standard Deviation; UPDRS, Unified Parkinson's Disease Rating Scale.

return to the starting position. Then they repeated the task at their own pace. The delay with which the light switched off was variable to ensure that the number of movements was identical in the three conditions.

2. Externally Cued (EC). The patients were required to "react and move as fast as possible in response to an auditory cue". When the green light switched off, subjects were instructed to return to their starting position.

3. Externally Cued-urgent (ECu). This condition was identical to the EC condition except that the auditory cue sounded as a ball was released at the top of the ramp (release mechanism was not visible). The patients were asked to stop this ball with the electromagnetic catch. Before each session, the subjects were told that they had to stop as many balls as possible. They were also told that they would be informed about their score at the end of the session. In agreement with this claim, feedback was given to the subjects at the end of each ECu session. Subjects were then told how many balls they missed (and stopped). The ramp tilt was adjusted for each patient before the experiment during a short training session so as to ensure a failure rate roughly equal to $50 \%$. In this latter condition, vision of the rolling ball was prevented by an opaque barrier and no rolling sound could be heard. ECu did only differ from EC by the sheer knowledge of a ball rolling. After completion of

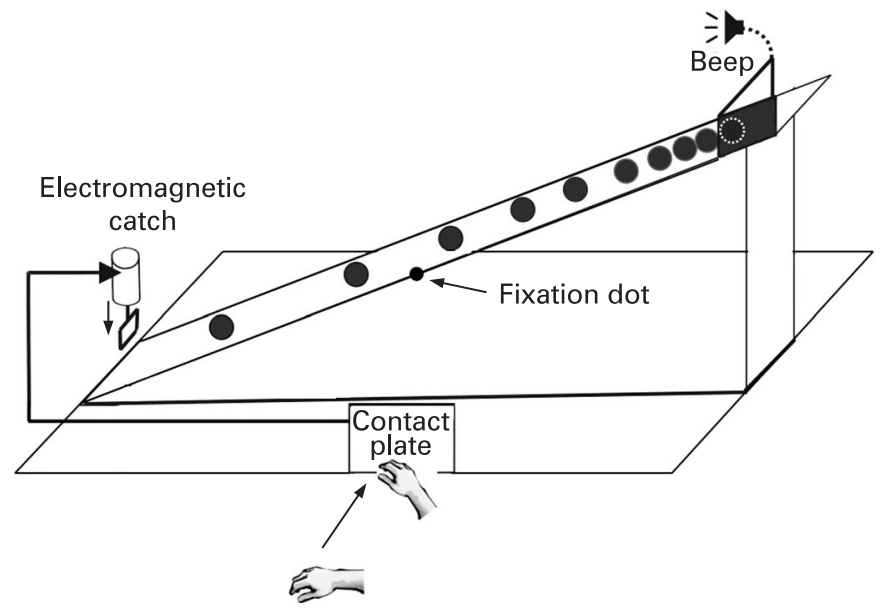

Figure 1 Experimental set-up. In the externally cued (EC) and externally cued-urgent (ECu) conditions, a beep cued the movement to the plate. In the ECu condition only, the patient had to press the plate before the ball had rolled out of the ramp. The ball could neither be seen nor heard. Ramp tilt was adjusted to ensure similar difficulty for each patient. the trial, the patients had to bring their hand back to the starting point after the light had switched off. The size of this starting point (microswitch) was small $(10 \times 5 \mathrm{~mm})$ and the patients had to perform this task under visual guidance. This prevented the occurrence of regular rhythmical movements in the self-generated condition. Each condition was performed during $100 \mathrm{sec}$ (15 movements) and was repeated four times in a randomised order, leading to a total number of 12 scans per subject.

\section{Behavioural analysis}

Movement duration ( $\mathrm{MD}$, interval from start position release to contact with the vertical plate) was computed for all conditions. Reaction time (RT, interval from beep to start position release) was computed for the externally triggered conditions. This parameter has no meaning for the internally driven condition. Two planned analyses were carried out using one-sided t-tests for dependent samples. First, the effect of external cueing was assessed by contrasting movement duration for the SI and EC conditions. Second, the effect of external cueing was assessed by contrasting movement duration and reaction time for the EC and ECu conditions. The threshold for statistical significance was set at 0.05 .

\section{PET data acquisition}

PET scanning was realised in the Neurology Hospital Imaging Centre (CERMEP, Lyon, France) on a CTI HR+ Siemens tomograph (CTI/Siemens, Knoxville, TN). The head was maintained in a fixed position using a moulded helmet. Head position was checked before and after each scan using a laser alignment together with reference points on the Reid's line.

A 10-min transmission scan was acquired using rotating rod sources filled with ${ }^{68} \mathrm{Ge} /{ }^{68} \mathrm{Ga}$. For each emission scan, $343 \pm 77$ $\mathrm{MBq}$ of $\mathrm{H}_{2}{ }^{15} \mathrm{O}$ were injected through a forearm catheter placed into the brachial vein. The integrated counts were collected for 90 seconds, starting 30 seconds after the injection. The interval between successive $\mathrm{H}_{2}{ }^{15} \mathrm{O}$ administrations was 9 min to allow for adequate radioactivity decay. Images were reconstructed by 3D back-filtered projection (Hanning filter; cut-off frequency, 0.5 cycles/pixel), giving a transaxial resolution of $6.5 \mathrm{~mm}$ full width at half maximum, and displayed in a $128 \times 128$ pixel format with 63 planes creating $2 \mathrm{~mm}$ cubic voxels.

\section{PET data analysis}

Using CAPP software, the original emission scans in ECAT7 file format were converted to ANALYZE file format that were then processed in MATLAB 5.3 (MathWorks, Natick, MA) using the Statistical Parametric Mapping software (SPM 99, Wellcome 
Department of Cognitive Neurology, MRC Cyclotron Unit, London, UK). In the first stage of analysis, the 12 images from each subject were realigned to the first scan with an automated algorithm for head movement correction and then normalised into the standard stereotactic space provided in SPM. The normalised images were smoothed with an isotropic Gaussian filter of $12 \mathrm{~mm}$ to account for variation in gyral anatomy and individual variability in structure-function relationships, and to improve the signal-to-noise ratio. Variations in global flow across subjects were removed by proportionally scaling each image to have an arbitrary level of $50 \mathrm{ml} / 100 \mathrm{ml} / \mathrm{min}$. Regional cerebral blood flow (rCBF) changes were statistically analysed for all voxels exceeding $80 \%$ of the mean value of the scan.

We analysed within-group modifications of $\mathrm{rCBF}$. The increase of $\mathrm{rCBF}$ related to the SI condition compared to the EC condition was assessed by SI-EC contrast. The increase of $\mathrm{rCBF}$ related to the $\mathrm{EC}$ condition compared with the SI condition was determined by EC-SI contrast. The increase of $\mathrm{rCBF}$ related to the motor urgency condition compared with the $\mathrm{EC}$ condition was derived from ECu-EC contrast.

We also analysed the covariation of $\mathrm{rCBF}$ with behavioural variables. ${ }^{15}$ In a first step, the mean $\mathrm{MD}$ and $\mathrm{RT}$ measured for each scan were used as covariates in a covariate-only model. For RT, we only selected the EC and ECu scans (RT has no meaning in the SI condition). For each scan, the mean RT was entered as a covariate. The total length of the covariate vector was 120 items (8 scans per subjects x 15 movements for each scan). For $\mathrm{MD}$, all the scans were selected (SI, Ec or Ecu). For each scan, the mean $\mathrm{MD}$ was entered as a covariate. The total length of the covariate vector was of 180 items (12 scans per subjects $\times 15$ movements for each scan). In a second step, the negative and positive covariations of $\mathrm{rCBF}$ with these covariates (RT and $\mathrm{MD}$ ) were determined independently of the condition. The statistical threshold was the same as the one used for the between-conditions contrasts (uncorrected p-value <0.001).

Finally, we compared the brain activation profile found in a previous study in normal subjects in the ECu condition ${ }^{15}$ with the one observed in patients with PD in the present work (ECu control - ECu patient; ECu patient - ECu control). Eight healthy subjects ( 4 males, 4 females; mean age $54 \pm 8.1$ years) were involved in this study. The experimental protocol and experimental apparatus were the same as the ones used in the present experiment.

Global differences in cerebral blood flow were covaried out for all brain voxels but, because of a priori hypothesis, small volume corrections were applied for all the aforementioned analysis for voxels located into a mask that included both frontal lobes, the ACC, parietal cortex, the cerebellum and basal ganglia-all regions that are known to be involved in motor function. ${ }^{8} 9$ 18-20 This mask was created using Matlab VoiTool (http://www.ihb. spb.ru/ pet_lab/VTO/VTOMain.html). The comparisons across conditions were made using $t$ statistics with appropriate linear contrasts, and then converted to $Z$-scores. Only voxels that exceeded a threshold of an uncorrected p-value $\leqslant 0.001$ were considered to be significant. The minimal cluster size comprised at least 10 voxels. All coordinates reported are based on the Talairach atlas ${ }^{21}$ and were derived from procedures developed by M. Brett (http://www.mrc-cbu.cam.ac.uk/ Imaging).

\section{RESULTS}

\section{Behavioural results}

\section{External cueing}

$\mathrm{MD}$ was significantly reduced when an external cue was present $(\mathrm{t}=2.30, \mathrm{p}<0.03)$. This variable decreased from $271 \mathrm{~ms}$ in SI to $209 \mathrm{~ms}$ in EC.

\section{Motor urgency}

RT was significantly reduced in the urgent condition $(t=2.82$, $p<0.02$ ). This variable decreased from $262 \mathrm{~ms}$ in EC to $234 \mathrm{~ms}$ in $\mathrm{ECu}$. The same trend was found for $\mathrm{MD}(\mathrm{t}=2.95, \mathrm{p}<0.02)$. This variable decreased from $209 \mathrm{~ms}$ in EC to $180 \mathrm{~ms}$ in ECu. These results are represented in figure 2 .

We did not find any significant correlation between the motor speed observed in urgent context (ECu-EC) and the duration of the disease (RT: $p>0.30 ; M D: p>0.95$ ) or the UPDRS score (RT: $p>0.25$; $M D: p>0.075$ ). These results remained unaffected when the atypical patient 1 (early disease onset and very long disease duration; table 1) was removed from the analyses.

\section{PET results}

Activation profile during SI versus EC movements

SI-related activity was found in the right lateral premotor cortex, DLPFC (BA 9 and 46), anterior cingulate (BA 32) cerebellar hemisphere and in the left medial frontal gyrus (BA 11
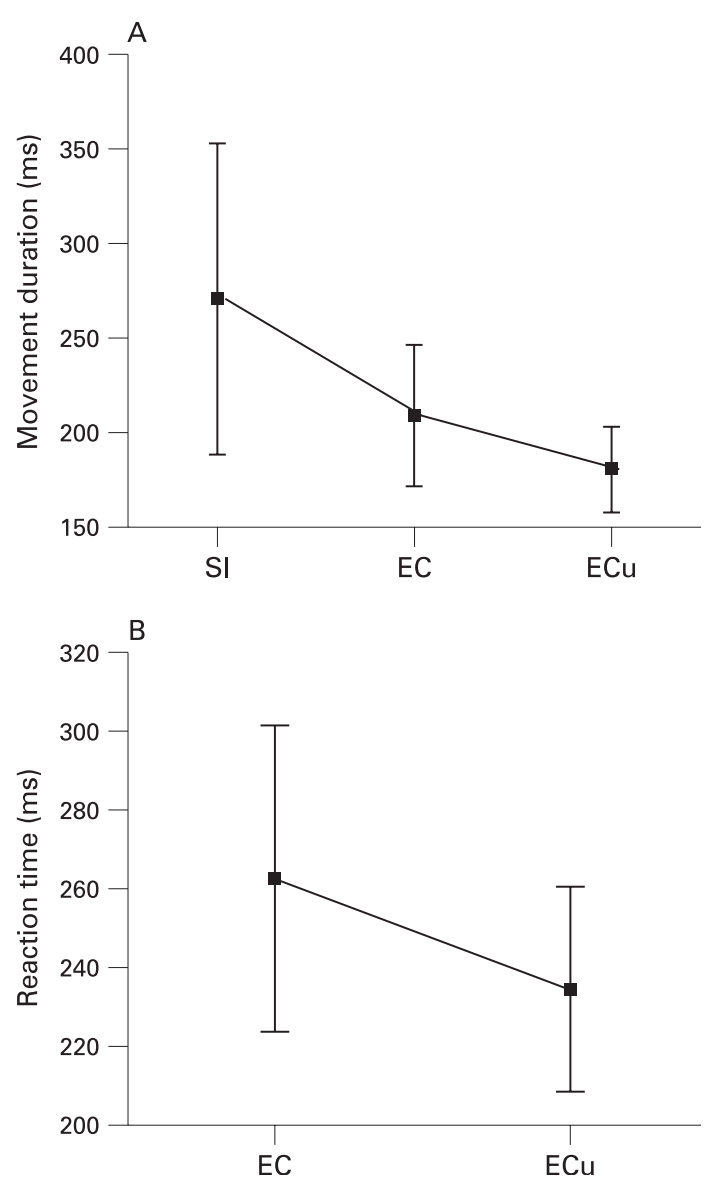

Figure 2 (A): Variations of movement duration as a function of the experimental conditions. (Symbols and error bars: mean $\pm S D$ ). (B): Variations of reaction time as a function of the experimental conditions. (Symbols and error bars: mean $\pm \mathrm{SD}$ ). EC, externally cued; ECu, externally cued-urgent; SI, self-initiated. 
Table 2 Significant rCBF increases in parkinsonian patients

\begin{tabular}{|c|c|c|c|c|c|c|}
\hline \multirow[t]{2}{*}{ Areas } & \multicolumn{5}{|c|}{ Stereotactic coordinates } & \multirow[t]{2}{*}{ Cluster size } \\
\hline & Left/right & $\mathrm{x}$ & $\mathrm{y}$ & $z$ & Z score & \\
\hline \multicolumn{7}{|l|}{$\mathrm{SI}>\mathrm{EC}$} \\
\hline \multirow[t]{2}{*}{ DLPFC (BA 9, 46) } & $\mathrm{R}$ & 22 & 47 & 37 & 3.70 & 192 \\
\hline & $\mathrm{R}$ & 41 & 41 & 12 & 3.12 & 200 \\
\hline Anterior cingulate cortex (BA 32) & $\mathrm{R}$ & 10 & 32 & 9 & 3.00 & 161 \\
\hline Primary motor cortex (BA 4) & $\mathrm{L}$ & -52 & 1 & 28 & 3.12 & 76 \\
\hline Superior parietal lobe (BA 7) & $\mathrm{L}$ & -31 & -69 & 45 & 3.09 & 220 \\
\hline \multicolumn{7}{|l|}{$E C>S I$} \\
\hline \multicolumn{7}{|l|}{ None } \\
\hline \multicolumn{7}{|l|}{$\mathrm{Ecu}>\mathrm{EC}$} \\
\hline Cerebellum (hemisphere) & $\mathrm{L}$ & -22 & -67 & -41 & 3.52 & 1700 \\
\hline
\end{tabular}

DLPFC, dorsolateral prefrontal cortex; EC, externally cued; ECu, externally cued-urgent; L, left; $R$, right; rCBF, regional cerebral blood flow; SI, self-initiated.

and 10), primary motor cortex (BA 4) and superior parietal lobe (BA 7). The location, coordinates and peak Z-scores of activated areas are detailed in table 2 .

Activation profile during EC versus SI movements

We did not observe significant $\mathrm{rCBF}$ increase when comparing the EC to the SI condition.

Activation profile during $\mathrm{ECu}$ versus $\mathrm{EC}$ condition

A significant activation foci was found in the left parasagittal cerebellar hemisphere (fig 3). At a slightly relaxed threshold $(z>2.67, p=0.001)$, we also observed an activation of the left DLPFC (BA 46) and anterior cingulate cortex (BA 32). The location, coordinates and peak Z-scores of activated areas are detailed in table 2 .

Compared with the normal subjects studied in a previous experiment, ${ }^{15}$ patients with $\mathrm{PD}$ had greater activations in the left superior frontal gyrus (BA 11), primary motor cortex (BA 4) and DLPFC (BA 9), as well as in the cerebellum bilaterally. No area of greater activation was found in the control subjects compared to the patients. These results are reported in table 3 .

Covariation of rCBF with behavioural measures

Movement duration scores showed a significant negative covariation with $\mathrm{rCBF}$ in the left parasagittal cerebellar hemisphere. This means that the shorter the movement time, the
Figure 3 Increased regional cerebral blood flow in the cerebellum in the externally cued-urgent condition compared with the externally cued conditions. Data are superimposed on transverse sections of a single subject brain MRI from SPM99. $p<0.001$ uncorrected (R: Right, L: Left).
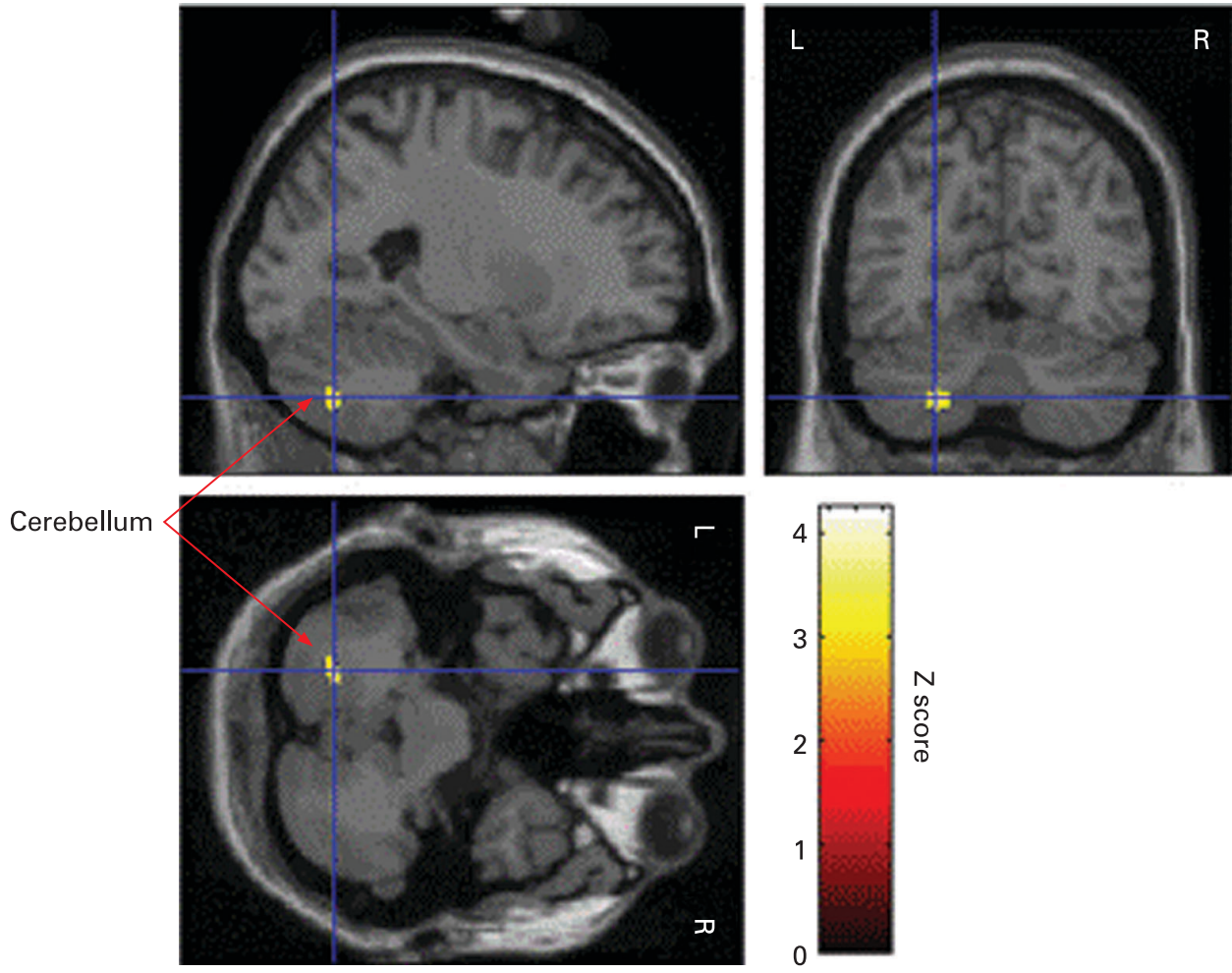
Table 3 Between-group analyses for the externally cued-urgent condition

\begin{tabular}{|c|c|c|c|c|c|}
\hline \multirow[b]{2}{*}{ Areas } & \multirow[b]{2}{*}{ Left/Right } & \multicolumn{3}{|c|}{ Stereotactic coordinates } & \multirow[b]{2}{*}{ Z score } \\
\hline & & $\mathbf{x}$ & y & $z$ & \\
\hline \multicolumn{6}{|l|}{ Controls $>P D$} \\
\hline None & & & & & \\
\hline \multicolumn{6}{|l|}{$P D>$ Controls } \\
\hline Cerebellum (Vermis) & $\mathrm{R}$ & 3 & -46 & -15 & 7.47 \\
\hline Cerebellum (Vermis) & $\mathrm{L}$ & -3 & -50 & -19 & 7.45 \\
\hline Superior frontal cortex (BA 11) & $\mathrm{L}$ & -4 & 57 & -18 & 6.13 \\
\hline Primary motor cortex (BA 4) & L & -55 & -15 & 38 & 5.64 \\
\hline DLPFC (BA 9) & $\mathrm{L}$ & -45 & 20 & 38 & 4.87 \\
\hline
\end{tabular}

All areas were significant at $p<0.05$ (corrected).

$\mathrm{BA}$, Brodmann area; DLPFC, dorsolateral prefrontal cortex.

greater the activation of this area. The location, coordinates and peak Z-scores of activated areas are detailed in table 4.

Reaction time showed a significant negative covariation with $\mathrm{rCBF}$ in the right $\mathrm{ACC}$ (BA 24) and the left primary motor cortex and superior frontal gyrus (BA 10). The location, coordinates and peak Z-scores of activated areas are detailed in table 4.

We did not find any significant correlation between the modulation of the cerebellar response and the severity (UPDRS motor score) or the duration of the disease.

\section{DISCUSSION}

\section{Behavioural data}

The present results indicate that: (1) Patients with PD can exceed their self-determined maximal movement speed for reaching in the context of externally cued conditions; (2) Patients with PD can exceed their externally driven maximal response speed in the context of a temporally pressing (or urgent) situation. These results are in agreement with previous behavioural studies showing that external cues and temporally constrained situations can improve bradykinesia in patients with PD . ${ }^{1-4} 142223$

\section{Changes of brain activation pattern according to motor context Externally cued versus self-initiated responses}

During the SI motor condition, patients with PD exhibited a much larger brain activation pattern than during the EC motor condition. This activation pattern concerned the right anterior cingulate, lateral premotor cortex and cerebellum, the left primary motor, prefrontal and superior parietal cortex and bilaterally in the prefrontal cortex. By contrast, no additional activation foci were observed in the EC compared with the SI condition. This latter finding is consistent with previous reports showing that SI and EC movements involve the same executive network. ${ }^{8} 924$
The additional activations observed in SI condition could reflect a compensatory process through which patients with $\mathrm{PD}$ try to overcome a specific difficulty to act in a self-determined mode, by recruiting areas that are known to be involved in the preparation, planning and selection of voluntary movement, such as the prefrontal and lateral premotor cortex..$^{918}$ On the other hand, the overactivation of the right cerebellum could reflect the involvement of the so-called "accessory motor pathways" that compensates for basal ganglia dysfunction in $\mathrm{PD}$ and has also been shown to include bilaterally the lateral premotor, primary motor and parietal cortex..$^{11}{ }^{132526}$ Finally, it is of interest to note the overactivation of the left primary motor cortex, indicating that patients with $\mathrm{PD}$ have to recruit motor preparation in a larger manner, but also motor execution areas due to the difficulties in performing SI movements. ${ }^{27}$

Interestingly, these overactivations observed in patients with PD were not associated with a normalisation of motor performance as the movement remained slower in the SI compared with the EC condition. Thus, it could be that this excess of brain activation could also reflect a lack of motor selectivity and would not be efficient. We could also hypothesise that the difficulties in performing the task induced a large recruitment of brain areas with, as a consequence, a slowdown of the movement. Similarly, in another study in PD on a working memory task, the large recruitment of brain areas was not associated with an improvement of motor performance and the rate of error was proportional to the importance of brain activation pattern. ${ }^{28}$

At first glance, the absence of activation within the basal ganglia network when the SI condition was compared with the EC condition seems at odds with the claim that the basal ganglia network plays a much more essential role in internally than in externally regulated actions. ${ }^{8} 924$ Direct support to this idea has been provided by functional imaging studies showing activation within the basal ganglia network for SI but not for externally driven movements. ${ }^{824}$ Interestingly, however, this

Table 4 Negative covariation of regional cerebral blood flow with the basic parameters of movement

\begin{tabular}{|c|c|c|c|c|c|c|}
\hline \multirow[b]{2}{*}{ Areas } & \multirow[b]{2}{*}{ Left/Right } & \multicolumn{3}{|c|}{ Stereotactic coordinates } & \multirow[b]{2}{*}{ Z score } & \multirow[b]{2}{*}{ Cluster size } \\
\hline & & $\mathbf{x}$ & $y$ & $\mathbf{z}$ & & \\
\hline \multicolumn{7}{|l|}{ Reaction time } \\
\hline Anterior cingulate cortex (BA 24) & $\mathrm{R}$ & 6 & 22 & -3 & 3.78 & 73 \\
\hline Primary motor cortex (BA 4) & $\mathrm{L}$ & -20 & -36 & 58 & 3.74 & 117 \\
\hline Superior frontal cortex (BA 10) & $\mathrm{L}$ & -11 & 53 & 20 & 3.29 & 29 \\
\hline \multicolumn{7}{|l|}{ Movement duration } \\
\hline Cerebellum (hemisphere) & $\mathrm{L}$ & -24 & -40 & -38 & 3.60 & 36 \\
\hline
\end{tabular}

All areas were significant at $p<0.001$ (uncorrected).

BA, Brodmann area. 
dissociation was only observed when the different movements types were compared to a rest condition. No higher activation in the basal ganglia network was reported when the self-initiated and externally driven movements were compared with each other. ${ }^{824}$ An absence of systematic involvement of the basal ganglia for internally regulated or self-initiated movements was also reported in electrophysiological and inactivation studies in monkeys. ${ }^{29-32}$ These data are consistent with the findings of the present study.

\section{Influence of urgency}

To explore the pattern of brain activation specific to motor urgency, we compared the ECu and EC conditions. We found that the movements executed in an urgent context were associated with a specific and restricted activation within the left (contralateral) cerebellum. This activation correlated with the movement speed observed at the behavioural level. No correlation was found between the modulation of the cerebellar response and the severity (UPDRS motor score) or the duration of the disease. This negative result is consistent with the hypothesis that the contralateral cerebellum is activated in urgent situations, irrespective of the disease. In agreement with this view, previous studies have shown that: (1) even if patients with PD are slower than healthy subjects, motor urgency speeds up the motor response by a similar amount in patients with PD and control subjects; ${ }^{14}(2)$ the contralateral cerebellar activation observed in the present experiment is also present in healthy subjects, when a similar experimental protocol is used. ${ }^{15}$ When considered together, these data strongly suggest that the contextual modulation of movement speed in urgent situations does not rely on the BG network.

Converging evidence has been provided, in primates and humans, that the parasagittal cerebellar hemisphere was involved in the control of movement velocity. In non-human primates, recording studies in the Purkinje cells of the lobules V and VI have demonstrated relations of single unit discharge to movement velocity. ${ }^{33}$ In healthy humans, imaging studies have shown velocity-related activity in the cerebellar lobule VI. ${ }^{35} 36$ In patients with cerebellar lesions or dysfunctions, movement velocity has been reported to decrease with respect to control subjects. ${ }^{37-39}$

In all the studies mentioned above, modulation of the movement velocity has been shown to involve the ipsilateral cerebellum. In the present study, the activation was identified in the contralateral hemisphere. As mentioned in previous reports, however, the contralateral cerebellum is often recruited in patients with $\mathrm{PD}^{10} 11$ and stroke ${ }^{40}$ to compensate for the pathological dysfunctions of the normal motor network. This structure is also activated, in healthy subjects, in urgent situations, when movement velocity needs to be "boosted". ${ }^{15}$ The cerebellar contralateral activation observed in the present study may serve the same purpose. It may be triggered by urgency and the need to increase movement velocity above a certain (standard) limit.

For the sake of completeness, it should be mentioned that recent imaging studies have linked the left cerebellum to attentional processes. ${ }^{42}{ }^{43}$ However, we do not think that this constitutes a convincing explanation of our results as the cerebellar attentional area has been located repeatedly, not in the parasagittal as in our case, but in the lateral part of the cerebellar hemisphere. ${ }^{42-44}$ However, we readily acknowledge the need for greater attentional control in the ECu condition, as suggested by the higher activation of the anterior cingulate cortex and left DLPFC in this condition compared with the EC condition.

Before bringing this discussion to an end, it may be worth mentioning that although urgency led to the recruitment of the left parasagittal cerebellum in both the patients and the controls, there were two important differences in the motor network recruited by these two populations in the $\mathrm{ECu}$ condition. First, patients with PD exhibited a larger level of activation in the cerebellar vermis and contralateral motor cortex. This pattern has been suggested to reflect a compensatory response for basal ganglia dysfunction. ${ }^{11}{ }^{13} \mathrm{M} 1$, in particular, is usually not underactivated in $\mathrm{PD}$, and several reports have shown that patients with $\mathrm{PD}$ tended to recruit this area more extensively than normal subjects to compensate for bradykinesia. ${ }^{11} 1325$ Second, the patients exhibited a greater activation of the prefrontal cortex (BA 11 and DLPFC). This region is involved in the preparation, planning and selection of voluntary movement. Accordingly, the overactivation of this latter in the ECu condition observed in patients with $\mathrm{PD}$ is clearly abnormal. However, recently, it has been suggested that some overactivations could reflect a facet of the primary pathophysiology of $\mathrm{PD}$, such as an inability to inhibit contextually inappropriate circuits. ${ }^{27}$

\section{CONCLUSION}

In summary, the present PET study shows a progression of brain activation from planning regions in the SI condition to more executive areas in the EC condition, with an additional contralateral cerebellar activation, in this condition, when the action is performed in an urgent context (ECu). The contralateral cerebellum is known to be a key node of the accessory motor circuitry typically recruited by patients with $\mathrm{PD}$ to compensate for basal ganglia dysfunction and by healthy subjects to increase movement velocity in urgent motor contexts. Interestingly, we did not find any evidence that PD affected the ability of the patients to increase the speed of their movements in urgent situations. This suggests that contextual modulations of movement speed do not implicate the BG circuitry.

Acknowledgements: This study has been supported by a grant from the Association France Parkinson.

Competing interests: None.

\section{REFERENCES}

1. Majsak MJ, Kaminski T, Gentile AM, et al. The reaching movements of patients with Parkinson's disease under self-determined maximal speed and visually cued conditions. Brain 1998;121:755-66.

2. Mazzoni P, Hristova A, Krakauer JW. Why don't we move faster? Parkinson's disease, movement vigor, and implicit motivation. J Neurosci 2007;27:7105-16.

3. Freeman JS, Cody FW, Schady W. The influence of external timing cues upon the rhythm of voluntary movements in Parkinson's disease. J Neurol Neurosurg Psychiatry 1993;56:1078-84.

4. Georgiou N, lansek R, Bradshaw JL, et al. An evaluation of the role of internal cues in the pathogenesis of parkinsonian hypokinesia. Brain 1993;116:1575-87.

5. Souques AA. Older description of parkinsonian persons who can run much easier than walk. Rev Neurol 1921;37:559-60.

6. van Donkelaar $\mathbf{P}$, Stein JF, Passingham RE, et al. Neuronal activity in the primate motor thalamus during visually triggered and internally generated limb movements. J Neurophysiol 1999;82:934-45.

7. van Donkelaar $\mathbf{P}$, Stein JF, Passingham RE, et al. Temporary inactivation in the primate motor thalamus during visually triggered and internally generated limb movements. J Neurophysiol 2000;83:2780-90.

8. Jahanshahi $\mathbf{M}$, Jenkins $I H$, Brown RG, et al. Self-initiated versus externally triggered movements. I. An investigation using measurement of regional cerebral blood flow with PET and movement-related potentials in normal and Parkinson's disease subjects. Brain 1995;118:913-33. 
9. Jenkins IH, Jahanshahi M, Jueptner $M$, et al. Self-initiated versus externally triggered movements. II. The effect of movement predictability on regional cerebral blood flow. Brain 2000;123:1216-28.

10. Rascol 0, Sabatini U, Fabre N, et al. The ipsilateral cerebellar hemisphere is overactive during hand movements in akinetic parkinsonian patients. Brain 1997;120:103-10.

11. Samuel M, Ceballos-Baumann AO, Blin J, et al. Evidence for lateral premotor and parietal overactivity in Parkinson's disease during sequential and bimanual movements. A PET study. Brain 1997;120:963-76.

12. Hanakawa T, Fukuyama $H$, Katsumi $Y$, et al. Enhanced lateral premotor activity during paradoxical gait in Parkinson's disease. Ann Neurol 1999;45:329-36.

13. Sabatini U, Boulanouar K, Fabre N, et al. Cortical motor reorganization in akinetic patients with Parkinson's disease: a functional MRI study. Brain 2000;123:394-403.

14. Ballanger B, Thobois S, Baraduc P, et al. "Paradoxical kinesis" is not a hallmark of Parkinson's disease but a general property of the motor system. Mov Disord 2006;21:1490-5.

15. Thobois S, Ballanger B, Baraduc $\mathrm{P}$, Le, et al. Functional anatomy of motor urgency. Neuroimage 2007;37:243-52.

16. Gibb WR, Lees AJ. The significance of the Lewy body in the diagnosis of idiopathic Parkinson's disease. Neuropathol Appl Neurobiol 1989;15:27-44.

17. Fahn S, Elton RL. UPDRS program members Parkinson's Disease Rating Scale. In: Fahn S, Marsden CD, Goldstein M, Calne DB, eds. Recent Developments in Parkinson's Disease. Florham Park: Macmillanm Healthcare Information, 1987:153163.

18. Deiber MP, Passingham RE, Colebatch JG, et al. Cortical areas and the selection of movement: a study with positron emission tomography. Exp Brain Res 1991;84:393402.

19. Deiber MP, Honda M, Ibanez V, et al. Mesial motor areas in self-initiated versus externally triggered movements examined with fMRl: effect of movement type and rate. J Neurophysiol 1999;81:3065-77.

20. Playford ED, Jenkins $I H$, Passingham RE, et al. Impaired mesial frontal and putamen activation in Parkinson's disease: a positron emission tomography study. Ann Neurol 1992;32:151-61.

21. Talairach J, Tournoux P. Co-Planar Stereotactic Atlas of the Human Brain. New York: Thieme Medical Publishers, 1988.

22. Morris ME, lansek R, Matyas TA, et al. Stride length regulation in Parkinson's disease. Normalization strategies and underlying mechanisms. Brain 1996;119:55168.

23. Azulay JP, Mesure S, Amblard B, et al. Visual control of locomotion in Parkinson's disease. Brain 1999;122:111-20.

24. Cunnington R, Windischberger C, Deecke L, et al. The preparation and execution of self-initiated and externally-triggered movement: a study of event-related fMRI. Neuroimage 2002;15:373-85.

25. Thobois S, Dominey P, Decety J, et al. Overactivation of primary motor cortex is asymmetrical in hemiparkinsonian patients. Neuroreport 2000;11:785-9.
26. Yu H, Sternad D, Corcos DM, et al. Role of hyperactive cerebellum and motor cortex in Parkinson's disease. Neuroimage 2007;35:222-33.

27. Turner RS, Grafton ST, McIntosh AR, et al. The functional anatomy of parkinsonian bradykinesia. Neuroimage 2003;19:163-79.

28. Mattay VS, Tessitore A, Callicott JH, et al. Dopaminergic modulation of cortical function in patients with Parkinson's disease. Ann Neurol 2002;51:156-64.

29. Mink JW, Thach WT. Basal ganglia motor control. I. Nonexclusive relation of pallidal discharge to five movement modes. J Neurophysiol 1991;65:273-300.

30. Kimura M, Aosaki T, Hu Y, et al. Activity of primate putamen neurons is selective to the mode of voluntary movement: visually guided, self-initiated or memory-guided. Exp Brain Res 1992;89:473-7.

31. Inase M, Buford JA, Anderson ME. Changes in the control of arm position, movement, and thalamic discharge during local inactivation in the globus pallidus of the monkey. J Neurophysiol 1996;75:1087-104.

32. Turner RS, Anderson ME. Context-dependent modulation of movement-related discharge in the primate globus pallidus. J Neurosci 2005;25:2965-76.

33. Coltz JD, Johnson MT, Ebner TJ. Cerebellar Purkinje cell simple spike discharge encodes movement velocity in primates during visuomotor arm tracking. J Neurosci 1999;19:1782-803.

34. Fu QG, Flament D, Coltz JD, et al. Relationship of cerebellar Purkinje cell simple spike discharge to movement kinematics in the monkey. J Neurophysiol 1997;78:478-91.

35. Turner RS, Grafton ST, Votaw JR, et al. Motor subcircuits mediating the control of movement velocity: a PET study. J Neurophysiol 1998;80:2162-76.

36. Turner RS, Desmurget M, Grethe J, et al. Motor subcircuits mediating the control of movement extent and speed. J Neurophysio/ 2003;90:3958-66.

37. Bonnefoi-Kyriacou B, Trouche E, Legallet $E$, et al. Planning and execution of pointing movements in cerebellar patients. Mov Disord 1995;10:171-8.

38. Straube A, Scheuerer W, Eggert T. Unilateral cerebellar lesions affect initiation of ipsilateral smooth pursuit eye movements in humans. Ann Neurol 1997;42:891-8.

39. Badescu RC, Lalonde R. Associations between a cerebellar motor dysfunction scale and movement initiation and completion in olivopontocerebellar atrophy. Rev Neurol 2001;157:1410-5

40. Hallett M. Plasticity of the human motor cortex and recovery from stroke. Brain Res Brain Res Rev 2001;36:169-74.

41. Desmurget $\mathbf{M}$, Bonnetblanc $\mathbf{F}$, Duffau $\mathrm{H}$. Contrasting acute and slow-growing lesions: a new door to brain plasticity. Brain 2007;130:898-914.

42. Allen G, Buxton RB, Wong EC, et al. Attentional activation of the cerebellum independent of motor involvement. Science 1997;275:1940-3.

43. Critchley HD, Elliott $R$, Mathias CJ, et al. Neural activity relating to generation and representation of galvanic skin conductance responses: a functional magnetic resonance imaging study. J Neurosci 2000;20:3033-40.

44. Mazoyer P, Wicker B, Fonlupt P. A neural network elicited by parametric manipulation of the attention load. Neuroreport 2002;13:2331-4. 